\title{
BMJ Open Prevalence of frailty and pain in hospitalised adult patients in an acute hospital: a protocol for a point prevalence observational study
}

\author{
Rosemary Saunders (D) , ${ }^{1}$ Kate Crookes (D) , ${ }^{1}$ Mustafa Atee (D) , ${ }^{2,3}$ \\ Caroline Bulsara (D) , ${ }^{4,5}$ Max K Bulsara (D) , ' Christopher Etherton-Beer (D) ,6,7 \\ Beverley Ewens (D) ," Olivia Gallagher (D) , ${ }^{1}$ Renee M Graham (D) , ${ }^{1}$ \\ Karen Gullick (D) , ${ }^{8}$ Sue Haydon, ${ }^{9}$ Kim-Huong Nguyen (D) , ${ }^{10}$ Bev O'Connell (D) , \\ Karla Seaman (D) , ${ }^{1}$ Jeff Hughes (D) ${ }^{2,11}$
}

To cite: Saunders $\mathrm{R}$, Crookes $\mathrm{K}$, Atee $\mathrm{M}$, et al. Prevalence of frailty and pain in hospitalised adult patients in an acute hospital: a protocol for a point prevalence observational study. BMJ Open 2021;11:e046138. doi:10.1136/ bmjopen-2020-046138

- Prepublication history for this paper is available online To view these files, please visit the journal online (http://dx.doi. org/10.1136/bmjopen-2020046138).

Received 26 0ctober 2020 Revised 16 February 2021 Accepted 16 February 2021

Check for updates

(C) Author(s) (or their employer(s)) 2021. Re-use permitted under CC BY-NC. No commercial re-use. See rights and permissions. Published by BMJ.

For numbered affiliations see end of article.

Correspondence to Dr Rosemary Saunders; rosemary.saunders@ecu.edu.au

\section{ABSTRACT}

Introduction Frailty and pain are associated with adverse patient clinical outcomes and healthcare system costs. Frailty and pain can interact, such that symptoms of frailty can make pain assessment difficult and pain can exacerbate the progression of frailty. The prevalence of frailty and pain and their concurrence in hospital settings are not well understood, and patients with cognitive impairment are often excluded from pain prevalence studies due to difficulties assessing their pain. The aim of this study is to determine the prevalence of frailty and pain in adult inpatients, including those with cognitive impairment, in an acute care private metropolitan hospital in Western Australia.

Methods and analysis A prospective, observational, single-day point prevalence, cross-sectional study of frailty and pain intensity of all inpatients (excluding day surgery and critical care units) will be undertaken. Frailty will be assessed using the modified Reported Edmonton Frail Scale. Current pain intensity will be assessed using the PainChek smart-device application enabling pain assessment in people unable to report pain due to cognitive impairment. Participants will also provide a numerical rating of the intensity of current pain and the worst pain experienced in the previous 24 hours. Demographic and clinical information will be collected from patient files. The overall response rate of the survey will be reported, as well as the percentage prevalence of frailty and of pain in the sample (separately for PainChek scores and numerical ratings). Additional statistical modelling will be conducted comparing frailty scores with pain scores, adjusting for covariates including age, gender, ward type and reason for admission.

Ethics and dissemination Ethical approval has been granted by Ramsay Health Care Human Research Ethics Committee WA/SA (reference: 2038) and Edith Cowan University Human Research Ethics Committee (reference: 2020-02008-SAUNDERS). Findings will be widely disseminated through conference presentations, peerreviewed publications and social media.

Trial registration number ACTRN12620000904976.

\section{Strengths and limitations of this study}

- This will be the first study to simultaneously assess the point prevalence of frailty and pain across all inpatients (excluding day surgery and critical care wards) in an acute hospital.

- The results of this study will inform future use of routine frailty assessment and a technology-driven assessment of pain.

- Technology-driven assessment of pain will enable pain intensity levels to be determined for patients who cannot verbalise their pain or provide a numerical pain rating and who therefore have previously been excluded from prevalence studies.

- Exclusion of critical care wards (ie, intensive care unit and coronary care unit) mean prevalence across the entire hospital cannot be fully determined.

\section{INTRODUCTION}

The ageing population and increasing number of frail older patients presents a challenge for health services. This patient group often have multiple co-morbidities and symptoms including pain. ${ }^{12}$ Frailty and pain can interact in such a way that symptoms of frailty such as cognitive decline can make pain difficult to assess, ${ }^{3}$ and pain can exacerbate the progression of frailty. ${ }^{4-6}$ Frailty and pain are both associated with negative clinical and patient outcomes, ${ }^{78}$ and significant costs to the healthcare system, ${ }^{9}{ }^{10}$ yet the prevalence of each and their occurrence in hospital settings are not well understood. ${ }^{1112}$

Frailty is defined as 'a medical syndrome with multiple causes and contributors that is characterised by diminished strength, endurance and reduced physiological function, that increases an individual's vulnerability for increased dependency and/or death' 
[Morley, p393]. ${ }^{13}$ Frailty is associated with increased risk of postoperative complications, increased length of hospital stay, functional dependency post-discharge, readmission to hospital and death during hospitalisation. ${ }^{814-16}$ One study identified an increase in healthcare costs of between $54 \%$ and $101 \%$ concomitant with increasing symptoms of frailty and costs of nursing care. ${ }^{9}$ Clinical Practice Guidelines recommend routine frailty screening for older adult patients. ${ }^{17}$

The difficulty in routinely assessing patients for frailty and determining its prevalence is in part due to the nature, design and administration of the assessment tools. Many of the assessment tools such as the phenotype mode ${ }^{18}$ are performance-based measures of physical function which are lengthy and require specialist administration. Recently, self-report measures of function have been developed, including the Reported Edmonton Frail Scale (REFS) ${ }^{19}$ and the modified REFS (mod-REFS), ${ }^{20}$ which have been shown to provide a valid estimate of frailty. ${ }^{19} 21$

A systematic review of community based cohort studies estimated $10.7 \%$ of the older adult population are categorised as frail..$^{22}$ Recent studies suggest the prevalence of frailty is higher for hospitalised older adults, reporting rates between $28 \%$ and $87 \% .{ }^{23-28}$ The large degree of variability may be, in part, due to differences in the methods of assessment of frailty, the lack of standardisation across studies, differences in the medical conditions and ward type included and variety of geographical locations. The focus on older adult patients and/or limitation to specific wards makes generalisation difficult and results do not provide a complete picture of the hospital-wide prevalence of frailty.

Only two studies were located that reported the point prevalence of frailty across all patients in an entire hospital. Richards $e t a l^{12}$ assessed frailty using the mod-REFS and found the prevalence to be $48.8 \%$ in a tertiary hospital in New Zealand. The prevalence of frailty was found to increase significantly with age and differences were found between admission types (eg, patients admitted to a medical specialty were frailer than those admitted to a surgical specialty $\left.{ }^{12}\right)$. Condon $e a^{29}$ assessed frailty using the Clinical Frail Scale ${ }^{30}$ and reported $52.2 \%$ prevalence of frailty in an acute university hospital in Ireland. Given the focus of the study was on incontinence, associations with frailty were not fully explored, however, increased frailty scores were associated with both increased urinary and faecal incontinence. ${ }^{29}$ Despite the difference in location (ie, New Zealand vs Ireland), these two studies suggest that approximately half of all inpatients within public hospitals are frail. However, the prevalence of frailty across all patients within an Australian hospital and within a private hospital setting has not previously been examined. This is an important area to investigate as private hospitals account for approximately $40 \%$ of all hospitalisations in Australia ${ }^{31}$ and there are key demographic differences between public and private hospitals (eg, private hospitals have a higher percentage of surgical compared with medical patients ${ }^{31}$; patients of a higher socioeconomic status than public hospitals ${ }^{31}$; and more patients aged 55-74 are treated in private hospitals than other age groups ${ }^{32}$ ) which may impact the prevalence of frailty or pain in the private hospital setting.

The prevalence of pain in hospital settings is also not clearly understood. Clinical assessment of pain is traditionally reliant on patient self-reports using numeric ratings of pain intensity on a $0-10$ scale $^{33}$ or pain scales developed for use with patients with a cognitive decline, such as the Abbey Pain Scale. ${ }^{34}$ Prevalence studies investigating pain in hospitals have used similar tools but the time frames over which patients are asked to rate their pain have varied (eg, current pain vs any pain in the last 24 hours). In a systematic review, Gregory and McGowan ${ }^{11}$ identified five previous point prevalence studies of current pain across entire hospitals ${ }^{35-39}$ that reported rates of between $37.7 \%$ and $84 \%$, and three studies of pain in the last 24 hours which reported rates between $52 \%$ and $65 \% .{ }^{35}{ }^{3640}$ Given the self-report nature of the commonly used tools, pain can be difficult to accurately assess in patients with cognitive impairment, meaning these patients are routinely excluded from pain prevalence studies. ${ }^{35-37}$ 39-42

In response to the problem of subjective pain assessment in cognitively impaired individuals, PainChek has been developed and validated as an effective, technology driven, multimodal, multiplatform and hybrid, pain assessment tool. PainChek is a smart-device application that evaluates pain in adults in 1 min using automated facial recognition and analysis to identify pain-related facial microexpressions, together with a series of user completed checklists of pain behaviours. ${ }^{43}{ }^{44}$ It uses the in-built cameras and processors of smart devices to assess the presence of pain-associated facial muscle movements using a 3 second video analysis of the individual's face. The application then guides the assessor through checklists of pain behaviours, which include items related to changes in vocalisations, movement, and behaviours enacted by individuals during the experience of pain (figure 1). The total of these behaviours informs the pain intensity according to the following categories: no pain (0-6), mild $(7-11)$, moderate $(12-15)$ and severe $(\geq 16) .{ }^{44}{ }^{45}$ Based on a number of clinical studies (total participants $\mathrm{n}=74,753$ paired pain assessments) the PainChek application has demonstrated sound psychometric validity and reliability and clinical utility in people with moderate-to-severe dementia. ${ }^{4-47}$ The prevalence of pain has not previously been assessed across an entire hospital using a technology driven assessment of pain. The use of such a device would allow data to be collected from all participants including those unable to provide a reliable self-report rating of their pain.

The aim of the proposed study is to conduct a point prevalence survey on frailty and pain of adult inpatients in an acute private hospital in Western Australia. The study will be the first worldwide to investigate the prevalence of frailty across the adult inpatients of an entire 

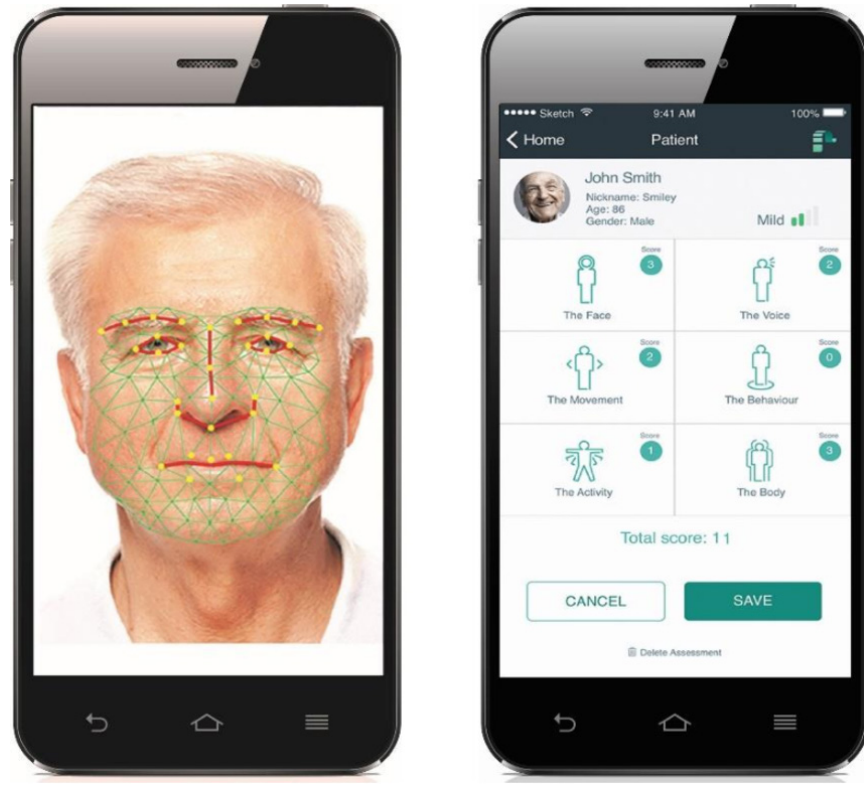

Figure 1 PainChek assess facial microexpressions that indicate the presence of pain. A pain intensity score is calculated across six domains of pain assessment. The face in this image is a royalty-free stock image freely available from: https://www.istockphoto.com/photo/studio-portrait-ofa-senior-man-gm157718744-21971579

acute private hospital (excluding patients in day surgery and critical care units), and the first across an entire Australian hospital. In addition, the proposed study will be the first point prevalence study of current pain across an entire hospital using a technology driven assessment of pain. Results of this study will provide an understanding of the distribution of frailty and pain and their concurrence across the hospital setting and guide future research into potential interventions.

\section{METHODS AND ANALYSIS}

\section{Study design and setting}

A prospective, observational, single-day point prevalence, cross-sectional study assessing frailty and pain will be undertaken. The study will be conducted across all non-critical care wards of an acute private metropolitan hospital in Perth, Western Australia. It is the largest private hospital in Western Australia with 738 licensed beds. The study will take place in late November 2020 . The Strengthening the Reporting of Observational Studies in Epidemiology Statement Checklist will be used to report the findings of the study. ${ }^{48}$

\section{Recruitment}

Potential participants will include all adult inpatients who were hospitalised at or before 08:00 hours on the day of data collection, excluding day procedure, intensive care (ICUs) and coronary care units (CCUs). It is anticipated there will be a maximum of 518 participants across 18 wards including surgical, medical, rehabilitation and mental health specialties. Participant inclusion and exclusion criteria are:

\section{Inclusion criteria}

- All acute care inpatients willing and able to provide informed verbal consent.

- All acute care inpatients who are unable to consent due to cognitive impairment or inability to verbally communicate, and have written proxy consent provided.

- Aged 18 years or over.

\section{Exclusion criteria}

The patient will not participate in the study if any of the following apply:

- Admitted to day procedures unit.

- Admitted to high-dependency areas (ICU, CCU).

- Hospitalised after 08:00 hours.

- Severe hearing impairment.

- Severe intellectual disability.

- Too unwell to participate.

- Refuse consent.

Excluded patients will be noted and the reason for exclusion recorded. If a patient or proxy who is declining to participate volunteers a reason for their refusal that reason will also be recorded.

Participant information sheets will be provided to inpatients in the 2 days prior to commencement of data collection. For patients admitted after 17:00 hours on the day prior to data collection the participant information sheet will be provided on the day of data collection.

Given the research is low risk, the interaction with the patient is minor, and assessments of pain and frailty are part of standard care, we will seek verbal consent from patients with their response recorded by the data collector. If a patient is unable to provide informed consent due to cognitive impairment or inability to verbally communicate, written proxy consent will be sought from their guardian or next-of-kin following guidelines from the Western Australian Department of Health to adhere to the requirements of the Western Australian Guardianship and Administration Amendment (Medical Research) Act $2020{ }^{49}$ On admission to the study site, patients are routinely assessed using the 4AT (Assessment Test) for delirium and cognitive impairment (4AT; www.the4at. com). For patients with a $4 \mathrm{AT}$ score of 4 or greater the data collector will check the patient's file for a cognitive assessment (either the Mini-Mental State Examination ${ }^{50}$ or Montreal Cognitive Assessment ${ }^{51}$ (MMSE, MoCA)). Where cognitive assessment indicates no cognitive impairment (MMSE $\geq 25$; MoCA $>25$ ) consent will be sought from the patient. Where cognitive assessment scores indicate severe cognitive impairment (MMSE $<18$; MoCA $<10$ ) proxy consent will be sought. Where cognitive assessment indicates mild-moderate cognitive impairment (MMSE 18-24; MoCA 10-25) or if no cognitive assessment has been performed the data collector will consult with the patient's medical team to determine if the patient has the 
capacity to consent or if proxy consent should be sought. If a patient is unable to speak English a telephone translation service will be used to interpret the information sheet, ask for consent and administer questionnaires.

\section{Data collection}

All data will be collected on a single day with approximately 15 min interactions with each participant. Data collectors will work in pairs, with one data collector conducting the pain and frailty assessments with the patient (or proxy where necessary), while the other will access the patient's file to record demographic and clinical information. Data collectors will be members of the research team, registered nurses from the study hospital and nursing students from the study university. All data collectors will be required to complete a 2-hour training session on the data collection protocol including the PainChek assessment no more than 7 days prior to data collection. Both the mod-REFS and PainChek were developed for use by non-health professionals. Evidence from previous studies found excellent inter-rater reliability, on the REFS when administered by two non-medically trained research scientists, ${ }^{19}$ and PainChek when administered by nurses trained to administer the assessment, ${ }^{46}$ which suggests a single assessment of each patient is sufficient.

On the day of data collection, before approaching patients, the data collectors will meet with the ward nurse manager to obtain a bed list of patients currently admitted to the ward. The nurse manager will identify patients too unwell to participate and those with an intellectual disability, who will be excluded from the study. Prior to approaching a patient, the data collectors will check the patient's 4AT score in the patient's file (score $<4$ patient will be approached, score $\geq 4$ cognitive assessments will be checked and proxy consent will be sought if required). If a patient is absent from their bed space, data collectors will return later in the day.

Each participant will have a pain and frailty assessment; demographic and clinical data will also be collected. During the pain and frailty assessments, data collectors will enter the participant's responses into a data collection form in Qualtrics running on an iPad or iPad mini. Where consent is sought from a proxy, the pain assessment will be conducted with the patient only using PainChek, and the frailty assessment will be completed by the proxy on behalf of the patient.

\section{Pain assessment}

Current pain status will be assessed at rest for all participants using the PainChek application on an iPad or iPad mini. PainChek uses a combination of automated facial recognition and other clinical indicators to calculate a pain intensity score. The score is based on the summation of 42 indicators of pain across 6 domains (ie, Face, Voice, Movement, Behaviour, Activity, Body) the presence of which are scored as 'yes' $=1$ and 'no' $=0$. As part of its multidiamentional design, PainChek also captures specific data on pain interference, which cover physical, emotional and social aspects of pain experience. PainChek scores will be stored in the PainChek cloud repository and entered into the Qualtrics data collection form.

Following the PainChek assessment participants who are able to self-report pain (ie, those without cognitive decline) will be asked to give a numerical rating of the intensity of their current pain and the worst pain they have experienced in the last 24 hours. These will be conducted using the routine verbal 0-10 Numerical Rating Scale where 0 is 'no pain at all' and 10 is 'worst possible pain'.

\section{Frailty assessment}

Frailty will be assessed using the mod-REFS which is a 13 -item self-report questionnaire scored from 0 to $18 .^{20}$ This tool was chosen as it is quick to administer $(<10 \mathrm{~min})$, can assess frailty prior to an acute illness, is validated for use in hospitals, ${ }^{20}$ and has previously been used to assess the point prevalence of frailty in a hospital setting. ${ }^{12}$ Participants will be asked two additional questions on their perceptions of their own frailty: (1) Do you consider yourself to be frail? (yes/no) and (2) Based on the answer to the last question, on a scale of $0-10$ where 0 is "not at all' and 10 is 'extremely' how frail do you think you are? In the case of participants requiring proxy consent due to cognitive impairment the mod-REFS and frailty questions will be completed by the proxy on behalf of the participant.

\section{Demographic and clinical data}

Routine admission data will be collected from the patient files including year of birth, gender, admission date, indigenous status, admission type (elective or acute) and admission mode (home or residential aged-care facility). Clinical information including admitting diagnosis, active medical conditions and analgesics (name, dose and time of last dose) will also be collected from patient files.

\section{Data reporting and analysis}

The overall response rate of the survey will be reported as a percentage of eligible patients. Reasons for exclusion and the associated percentages will also be reported. The distributions of demographic and clinical characteristics will be described for the overall cohort using standard summary statistics.

The percentage prevalence of frailty in the sample will be calculated based on the mod-REFs score where a score of 8 and above is classified as frail, and will also be calculated in terms of the severity of frailty for the classifications: not frail $(0-5)$, apparently vulnerable $(6-7)$, mild frailty (8-9), moderate frailty $(10-11)$ and severe frailty (12-18) ${ }^{20}$ Prevalence will be reported across the whole sample and by specialty (ie, medical, surgical, rehabilitation, mental health). Comparison between scores on the mod-REFS and subjective perceptions of personal frailty obtained from supplementary questions will be explored (ie, do patients classified as severely frail on the modREFS classify themselves as frail more often, and rate their frailty higher, than those classified as moderately 
frail). Demographics for frail vs not frail patients will be reported and explored.

Percentage prevalence of pain across the whole sample and by specialty will be calculated separately for PainChek scores ( $\leq 6$ no pain, $\geq 7$ pain), ${ }^{45}$ numerical ratings of current pain ( 0 no pain, $\geq 1$ pain) and numerical ratings of worst pain in the last 24 hours ( 0 no pain, $\geq 1$ pain). Demographic characteristics of the patients reporting pain vs no pain will be described and explored. Prevalence will also be explored in terms of intensity of pain for PainChek (0-6 no pain, 7-11 mild, 12-15 moderate and $\geq 16$ severe $)^{44}$ and the numerical ratings (1-4 mild, 5-6 moderate, $7-10$ severe) ${ }^{52}$

In accordance with the intention-to-treat principle, all available data from all participants will be included in the primary statistical analysis. The analysis will involve the use of regression models to identify important factors related to the prevalence of frailty and pain reporting point estimates and $95 \%$ CIs along with a $p$ value of 0.05 to determine the statistical significance of important risk factors. Analyses will be conducted using Stata V.16 IC. ${ }^{53}$

\section{Data management}

Data will be managed according to the Australian National Statement on Ethical Conduct in Human Research (2007). No identifying data will be collected. Participants will be assigned a participant number based on their ward and bed number. Hard copy data collection forms will be stored in locked filing cabinets at the university accessible only by research team members. The data and participant information collected will be stored securely on University servers only accessible by research team members on password protected computers. PainChek data will be stored in a repository within the PainChek secure cloud database. Data will only be accessible by the research team members via a password protected Web Administration Portal. Data may be made available to other researchers for future research and may be published online according to publisher's requirements. All data will be destroyed after 7 years.

\section{Patient and public involvement}

Patients at the site are represented by a consumer representative from the hospital consumer advisory board. The consumer representative was invited to be part of the project at the commencement as a member of the research team and was involved in the study design. The research questions were discussed with the team and the proposal that included the research questions and outcome measures was reviewed by the team.

As we will not be collecting any personal or contact information from the study participants, it will not be possible to disseminate the research findings to them directly. However, findings will be communicated via the hospital newsletter and national hospital group newsletter and the PainChek website. The consumer representative will be involved in the discussions about the dissemination plan.

\section{ETHICS AND DISSEMINATION}

Given the relationship between cognitive decline and frailty it is important to include patients with cognitive impairment in this study. Similarly, in order to measure prevalence across the entire hospital it is important to include inpatients admitted to the mental health wards. The inclusion of participants with cognitive impairment and mental illness requires particular consideration of the consent procedures and risk of distress for these patients as per the Australian National Statement on Ethical Conduct in Human Research (2007). Patients in the mental health wards of the study hospital are all admitted voluntarily and can provide informed consent. Any refusal to participate will be respected. Where cognitive impairment means a patient is unable to provide informed consent, consent will be sought from the patient's legal guardian or next-of-kin. If at any point a patient exhibits signs of distress, the assessment will cease, and nursing assistance will be sought.

This study has ethical approval from both the Ramsay Health Care Human Research Ethics Committee for Western Australia and South Australia (reference number: 2038) and the Edith Cowan University Human Research Ethics Committee (reference: 2020-02008-SAUNDERS).

Results of this study will be disseminated at a number of levels. A report of the study findings will be provided to the study hospital and may be used to review its procedures and to guide a larger prevalence study across all hospitals run by the operator in Australia. Regardless of the outcomes, it is our intention to publish the results of this study in a peer-reviewed journal. Findings will also be presented at Australian and international conferences. Publications resulting from this study will be publicised through project and departmental social media.

\section{Author affiliations}

${ }^{1}$ Centre for Research in Aged Care, School of Nursing \& Midwifery, Edith Cowan University, Joondalup, Western Australia, Australia

${ }^{2}$ School of Pharmacy and Biomedical Sciences, Curtin University, Bentley, Western Australia, Australia

${ }^{3}$ The Dementia Centre, St Leonards, New South Wales, Australia

${ }^{4}$ School of Nursing and Midwifery, University of Notre Dame Australia, Fremantle, Western Australia, Australia

${ }^{5}$ Institute for Health Research, The University of Notre Dame Australia, Fremantle, Western Australia, Australia

${ }^{6}$ School of Medicine and Pharmacology, University of Western Australia, Crawley, Western Australia, Australia

${ }^{7}$ WA Centre for Health \& Ageing, University of Western Australia, Crawley, Western Australia, Australia

${ }^{8}$ Clinical Services, Hollywood Private Hospital, Nedlands, Western Australia, Australia ${ }^{9}$ Clinical Services, Hollywood Private Hospital, Nedlands 6009, Western Australia, Australia

${ }^{10}$ Faculty of Medicine and Biomedical Sciences, The University of Queensland, Saint Lucia, Queensland, Australia

${ }^{11}$ PainChek Ltd, Bentley, Western Australia, Australia

Twitter Kate Crookes @KateCrookes and Renee M Graham @rgrahamresearch

Acknowledgements We would like to acknowledge and thank the management and staff of Hollywood Private Hospital for their enthusiastic cooperation and assistance with this study.

Contributors The study was designed by all authors (RS, MA, CB, MKB, CE-B, BE, $\mathrm{OG}, \mathrm{RMG}, \mathrm{KG}, \mathrm{KN}, \mathrm{BO}, \mathrm{KS}$ and $\mathrm{JH}$ ) with contribution from SH. KC and RS drafted the 
manuscript and all authors have reviewed and approved the final manuscript. SH is a consumer representative from Hollywood Private Hospital.

Funding This work is supported by the Ramsay Hospital Research Foundation.

Competing interests KG is the Director of Clinical Services at Hollywood Private Hospital. Professor Jeff Hughes and Mr Mustafa Atee are two of the originators PainChek, which is marketed by PainChek. Both have shareholdings in PainChek Ltd (ASX:PCK), which is a publicly listed company in the Australian Share Securities. They have a granted patent entitled 'A pain assessment method and system" (PCT/ AU2015/000501) in Australia, Japan, China and the USA. JH holds the position of Chief Scientific Officer of PainChek while serving as a Professor at the School of Pharmacy and Biomedical Sciences, Curtin University. MA formerly served (October 2018-May 2020) as a Research Scientist for PainChek. He is also PhD Candidate with the School of Pharmacy and Biomedical Sciences, Curtin University.

Patient and public involvement Patients and/or the public were involved in the design, or conduct, or reporting, or dissemination plans of this research. Refer to the Methods section for further details.

Patient consent for publication Not required.

Provenance and peer review Not commissioned; externally peer reviewed.

Open access This is an open access article distributed in accordance with the Creative Commons Attribution Non Commercial (CC BY-NC 4.0) license, which permits others to distribute, remix, adapt, build upon this work non-commercially, and license their derivative works on different terms, provided the original work is properly cited, appropriate credit is given, any changes made indicated, and the use is non-commercial. See: http://creativecommons.org/licenses/by-nc/4.0/.

\section{ORCID iDs}

Rosemary Saunders http://orcid.org/0000-0001-6213-4694

Kate Crookes http://orcid.org/0000-0001-6887-6197

Mustafa Atee http://orcid.org/0000-0002-0837-3245

Caroline Bulsara http://orcid.org/0000-0003-4482-563X

Max K Bulsara http://orcid.org/0000-0002-8033-6123

Christopher Etherton-Beer http://orcid.org/0000-0001-5148-0188

Beverley Ewens http://orcid.org/0000-0003-2008-7214

Olivia Gallagher http://orcid.org/0000-0002-0023-0039

Renee M Graham http://orcid.org/0000-0001-8725-0296

Karen Gullick http://orcid.org/0000-0001-9503-442X

Kim-Huong Nguyen http://orcid.org/0000-0002-2592-9372

Bev 0'Connell http://orcid.org/0000-0001-9733-5218

Karla Seaman http://orcid.org/0000-0003-4611-9616

Jeff Hughes http://orcid.org/0000-0001-5204-0870

\section{REFERENCES}

1 Ardoino I, Franchi C, Nobili A, et al. Pain and frailty in hospitalized older adults. Pain Ther 2020;9:727-40.

2 Lin T, Zhao Y, Xia X, et al. Association between frailty and chronic pain among older adults: a systematic review and meta-analysis. Eur Geriatr Med 2020;11:945-59.

3 Frampton M. Experience assessment and management of pain in people with dementia. Age Ageing 2003;32:248-51.

4 Niederstrasser NG, Rogers NT, Bandelow S. Determinants of frailty development and progression using a multidimensional frailty index: evidence from the English longitudinal study of ageing. PLoS One 2019;14:1-16.

5 Saraiva MD, Suzuki GS, Lin SM, et al. Persistent pain is a risk factor for frailty: a systematic review and meta-analysis from prospective longitudinal studies. Age Ageing 2018;47:785-93.

6 Wade KF, Marshall A, Vanhoutte B, et al. Does pain predict frailty in older men and women? findings from the English longitudinal study of ageing (ELSA). J Gerontol A Biol Sci Med Sci 2017;72:403-9.

7 Lohman MC, Whiteman KL, Greenberg RL, et al. Incorporating persistent pain in phenotypic frailty measurement and prediction of adverse health outcomes. J Gerontol A Biol Sci Med Sci 2017;72:216-22.

8 Dent E, Chapman I, Howell S, et al. Frailty and functional decline indices predict poor outcomes in hospitalised older people. Age Ageing 2014;43:477-84.

9 Hajek A, Bock J-O, Saum K-U, et al. Frailty and healthcare costslongitudinal results of a prospective cohort study. Age Ageing 2018;47:233-41.

10 Gaskin DJ, Richard P. The economic costs of pain in the United States. J Pain 2012;13:715-24.
11 Gregory J, McGowan L. An examination of the prevalence of acute pain for hospitalised adult patients: a systematic review. J Clin Nurs 2016;25:583-98.

12 Richards SJG, D'Souza J, Pascoe R, et al. Prevalence of frailty in a tertiary Hospital: a point prevalence observational study. PLoS One 2019;14:e0219083

13 Morley JE, Vellas B, van Kan GA, et al. Frailty consensus: a call to action. J Am Med Dir Assoc 2013;14:392-7.

14 Kahlon S, Pederson J, Majumdar SR, et al. Association between frailty and 30-day outcomes after discharge from hospital. CMAJ 2015;187:799-804.

15 Khandelwal D, Goel A, Kumar U, et al. Frailty is associated with longer hospital stay and increased mortality in hospitalized older patients. J Nutr Health Aging 2012;16:732-5.

16 Lin H-S, Watts JN, Peel NM, et al. Frailty and post-operative outcomes in older surgical patients: a systematic review. BMC Geriatr 2016;16:157.

17 Dent E, Lien C, Lim WS, et al. The Asia-Pacific clinical practice guidelines for the management of frailty. J Am Med Dir Assoc 2017;18:564-75.

18 Fried LP, Tangen CM, Walston J, et al. Frailty in older adults: evidence for a phenotype. J Gerontol A Biol Sci Med Sci 2001;56:M146-57.

19 Hilmer SN, Perera V, Mitchell S, et al. The assessment of frailty in older people in acute care. Australas J Ageing 2009;28:182-8.

20 Rose M, Yang A, Welz M, et al. Novel modification of the reported Edmonton frail scale. Australas J Ageing 2018;37:305-8.

21 Alexander NB, Guire KE, Thelen DG, et al. Self-Reported walking ability predicts functional mobility performance in frail older adults. $J$ Am Geriatr Soc 2000;48:1408-13.

22 Collard RM, Boter $\mathrm{H}$, Schoevers RA, et al. Prevalence of frailty in community-dwelling older persons: a systematic review. J Am Geriatr Soc 2012;60:1487-92.

23 Chong E, Ho E, Baldevarona-Llego J, et al. Frailty and Risk of Adverse Outcomes in Hospitalized Older Adults: A Comparison of Different Frailty Measures. J Am Med Dir Assoc 2017;18:638.e7-638. e11.

24 Dorner TE, Luger E, Tschinderle J. Association between nutritional status (MNA $®-\mathrm{SF}$ ) and frailty (SHARE-FI) in acute hospitalised elderly patients. J Nutr Heal Aging 2014;18:264-9.

25 Hammami S, Zarrouk A, Piron C, et al. Prevalence and factors associated with frailty in hospitalized older patients. BMC Geriatr 2020;20:144

26 Hao Q, Zhou L, Dong B, et al. The role of frailty in predicting mortality and readmission in older adults in acute care wards: a prospective study. Sci Rep 2019;9:1207.

27 Joosten E, Demuynck M, Detroyer E, et al. Prevalence of frailty and its ability to predict in hospital delirium, falls, and 6-month mortality in hospitalized older patients. BMC Geriatr 2014;14:1.

28 Hewitt J, Moug SJ, Middleton M, et al. Prevalence of frailty and its association with mortality in general surgery. Am J Surg 2015;209:254-9.

29 Condon M, Mannion E, Molloy DW, et al. Urinary and faecal incontinence: point prevalence and predictors in a university hospital. Int J Environ Res Public Health 2019;16:194.

30 Rockwood K, Song X, MacKnight C, et al. A global clinical measure of fitness and frailty in elderly people. CMAJ 2005;173:489-95.

31 Australian Institute of Health and Welfare. Australia's hospitals at a glance 2018-2019, 2020.

32 Australian Institute of Health and Welfare. Australian hospital statistics 2011-2012, 2013. Available: www.aihw.gov.au

33 Daut RL, Cleeland CS, Flanery RC. Development of the Wisconsin brief pain questionnaire to assess pain in cancer and other diseases. Pain 1983;17:197-210.

34 Abbey J, Piller N, De Bellis A, et al. The Abbey pain scale: a 1-minute numerical indicator for people with end-stage dementia. Int $J$ Palliat Nurs 2004;10:6-13.

35 Melotti RM, Samolsky-Dekel BG, Ricchi E, et al. Pain prevalence and predictors among inpatients in a major Italian teaching hospital. A baseline survey towards a pain free Hospital. Eur J Pain 2005;9:485-95.

36 Costantini M, Viterbori P, Flego G. Prevalence of pain in Italian hospitals: results of a regional cross-sectional survey. $J$ Pain Symptom Manage 2002;23:221-30.

37 Trentin L, Visentin M, de Marco R. Prevalence of pain in a public Hospital: correlation between patients and caregivers. $J$ Headache Pain 2001;2:73-8.

38 Sawyer J, Haslam L, Robinson S, et al. Pain prevalence study in a large Canadian teaching hospital. Pain Manag Nurs 2008;9:104-12. 
39 Sawyer J, Haslam L, Daines P. Pain prevalence study in a large Canadian teaching hospital. Round 2: lessons learned? Pain Manag Nurs 2010;11:45-55

40 Wadensten B, Fröjd C, Swenne CL, et al. Why is pain still not being assessed adequately? results of a pain prevalence study in a university hospital in Sweden. J Clin Nurs 2011;20:624-34.

41 Das SK, Dhar SS, Panigrahi A. Prevalence of pain and its characteristics in hospitalized patients in an Indian teaching hospital. Pain Manag Nurs 2020;21:299-303.

42 Xiao $\mathrm{H}$, Liu H, Liu J, et al. Pain prevalence and pain management in a Chinese Hospital. Med Sci Monit 2018;24:7809-19.

43 Atee M, Hoti K, Hughes JD. A technical note on the PainChek ${ }^{\mathrm{TM}}$ system: a web portal and mobile medical device for assessing pain in people with dementia. Front Aging Neurosci 2018;10:117.

44 Atee M, Hoti K, Parsons R, et al. Pain assessment in dementia: evaluation of a point-of-care technological solution. J Alzheimers Dis 2017;60:137-50.

45 Hoti K, Atee M, Hughes JD. Clinimetric properties of the electronic pain assessment tool (ePAT) for aged-care residents with moderate to severe dementia. J Pain Res 2018;11:1037-44.

46 Atee M, Hoti K, Hughes JD. Psychometric evaluation of the electronic pain assessment tool: an innovative instrument for individuals with moderate-to-severe dementia. Dement Geriatr Cogn Disord 2017;44:256-67.

47 Atee M, Hoti K, Parsons R, et al. A novel pain assessment tool incorporating automated facial analysis: interrater reliability in advanced dementia. Clin Interv Aging 2018;13:1245-58.

48 von Elm E, Altman DG, Egger M, et al. Strengthening the reporting of observational studies in epidemiology (STROBE) statement: guidelines for reporting observational studies. BMJ 2007;335:806-8.

49 Parliament of Western Australia. Guardianship and administration Amendment (medical research) act 2020, 2020. Available: https:// www.legislation.wa.gov.au/legislation/statutes.nsf/law_a147258.htm

50 Folstein MF, Folstein SE, McHugh PR. "Mini-mental state". A practical method for grading the cognitive state of patients for the clinician. J Psychiatr Res 1975;12:189-98.

51 Nasreddine ZS, Phillips NA, Bédirian V, et al. The Montreal cognitive assessment, MoCA: a brief screening tool for mild cognitive impairment. J Am Geriatr Soc 2005;53:695-9.

52 Serlin RC, Mendoza TR, Nakamura Y, et al. When is cancer pain mild, moderate or severe? grading pain severity by its interference with function. Pain 1995:61:277-84.

53 StataCorp. Stata statistical software: release 16, 2019. 